See discussions, stats, and author profiles for this publication at: https://www.researchgate.net/publication/328167893

\title{
Space Robot Teleoperation Experiment and System Evaluation Method
}

Conference Paper · May 2018

DOI: 10.1109/MCEC.2018.8469654

3 authors, including:

D. Guanhua Feng

Chinese Academy of Sciences

7 PUBLICATIONS 2 CITATIONS

SEE PROFILE

Some of the authors of this publication are also working on these related projects:

Project Underwater Vehicle View project 


\title{
Space Robot Teleoperation Experiment and System Evaluation Method
}

\author{
Guanhua Feng ${ }^{\dagger}$, Wenhao Li ${ }^{\dagger \ddagger *}$ and Heng Zhang ${ }^{\dagger \ddagger}$ \\ ${ }^{\dagger}$ Institute of Mechanics, Chinese Academy of Sciences \\ No.15 Beisihuanxi Road, Beijing, P.R.China 100190

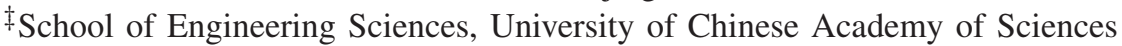 \\ No.19(A) Yuquan Road, Shijingshan District, Beijing, P.R.China 100049 \\ *Email: liwenhao@imech.ac.cn (Corresponding author)
}

\begin{abstract}
This paper focuses on the topic of teleoperation experiment and system evaluation for space robot. First, characteristics and key techniques of Space teleoperation system has been analyzed, and ten kinds of universal capabilities were summed up for a complete space teleoperation system based on its tele-operated, tele-scene, tele-system and tele-cooperative characteristics. Second, for each capability its detailed functional and/or quantitative evaluation items were summarized, and 92 functional items and 49 quantitative items covering all capabilities were listed for comprehensive teleoperation system evaluation. Then teleoperation experiment and system evaluation method was proposed and interpreted based on evaluation items. Finally, through the design of evaluation unit and example the effectiveness of the proposed method was validated.

Keywords-teleoperation experiment and system evaluation; characteristics; universal capabilities; evaluation items; evaluation method
\end{abstract}

\section{INTRODUCTION}

Space robot and its teleoperation, the combination product of aerospace engineering and robotics science, are important approaches to assist ground crew or replace astronauts, in space operations, to perform dangerous or long-term space missions [1]. They enhance the human ability to explore the space environment, which plays an important role in large space activities such as space shuttle and international space station [2].

Space robot teleoperation system, as the core system for the control of space robot by operators, makes full use of decision intelligence, information resources and high-performance devices support from the operations center to enhance the operational safety and functional integrity of space robot while improving the tasks execution efficiency, which has an irreplaceable role in the development of space robotics technologies. Therefore, aerospace powers in the world are all actively engaged in the research on space robots and their teleoperation systems [3]-[7].

The teleoperation system now is usually designed only for the specific task of the operation object and has great differences in structure, function, performance and implementation depending on the its missions. Currently, there is a lack of a unified evaluation system and no uniform standard for its evaluation methods. In this paper, we will study the evalu- ation methods of a complete teleoperation system and good teleoperation experiment for space robots and their missions.

\section{Evaluation Method}

As shown in Fig. 1, we first give the evaluation method directly for a space robot teleoperation system and its experiment. From the folw chart we know, experiment of specific $\operatorname{task}(\mathrm{s})$ is evaluated and its statistical results are used for the further teleoperation system evaluation. Then detailed steps of the proposed method are interpreted in further. In section II-A, we introdue the characteristic classification of a teleoperation system. Based on the interpretation of system characteristics, section II-B summarizes characteristics expansion to ten kinds of universal capabilities of a complete teleoperation system. The next section II-C discusses the evaluation method for teleoperation experiment. The last section treats II-D teleoperation system evaluation method.

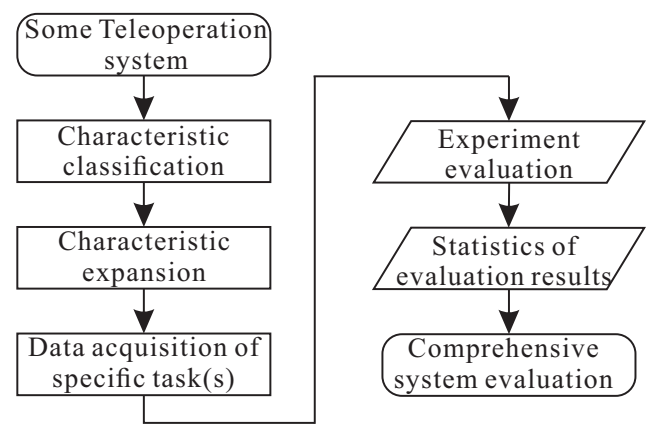

Fig. 1. Teleoperation experiment and system evaluation method

\section{A. Characteristic Classification}

The development of teleoperation system is mainly for specific task at present, and required capabilities and evaluation contents of teleoperation operation are not the same for different tasks. But due to the remote operation and task diversity, the main characteristics of a complete teleoperation system include the following four.

1) Tele-operated Characteristic: From the perspective of mission requirements, the main task of teleoperation system is to make field equipment work as expected. To achieve this goal, teleoperation system should have the tele-operated 
characteristic. The tele-operated characteristic is operability including operation integrity, real-time operation, operation safety, operation autonomy, operation backup/reproduction, operation efficiency; and its main indexes are coverage capability of operation mode to teleoperation task, elapsed time for processing field information and operation instructions by teleoperation system, security handling and coverage capability of teleoperation system for abnormal conditions, operator participation in the process of remote field feedback and remote instructions processing, operation convenience of teleoperation, and reflection intuitiveness of remote field information.

2) Tele-scene Characteristic: From the perspective of physical environment, the teleoperation system is a remote operation system. To accomplish teleoperation task effectively and smoothly, teleoperation system must feeds the remote field state in a intuitive form back to operator in teleoperation system. So the teleoperation system should have telescene characteristic. Tele-scene characteristic mainly include the sufficiency, authenticity and real-time of field state and field equipment response feedback, continuity, intuitiveness and time delay of field information; and its main indexes are coverage degree of feedback information to key indicator information of field state, bit error rate, refresh rate and fine degree of feedback information, time delay effect reduction capability of teleoperation system for field state processing, and prediction accuracy after time delay reduction.

3) Tele-system Characteristic: As the remote operation node of teleoperation task, the teleoperation system should have tele-system characteristic including appearance, equipment integration, electromechanical performance, environment adaptability, software/hardware operation stability, supporting facilities completeness of teleoperation system; and its main indexes are teleoperation system integration, working environment range of electricity, gas and temperature, capability of over-voltage protection, power outage protection and electromagnetic protection, continuous and stable working time of software/hardware, and supporting equipment and softwares.

4) Tele-cooperative Characteristic: When the teleoperation system is connected to operation network and cooperates with other teleoperation systems, it has become a operation terminal of the teleoperation network. It can be classified, by the number of operation terminal and object, to one-operator-multi-robot, multi-operator-one-robot, multioperator-multi-robot, and mixed operation of the above three. There is information exchange among teleoperation terminals and objects. The tele-cooperative characteristic is mainly reflected in tolerance to teleoperation terminal, synchronization of cooperative operation, and autonomy and security protection capability of data processing in cooperative operation.

\section{B. Characteristic Expansion}

1) Universal Capabilities of Complete Teleoperation System: Based on the main system characteristics above, ten kinds of universal universal capabilities of a complete teleoperation system can be expanded as shown in Fig. 2.
2) Evaluation Items for Universal Capabilities: In section II-B1, where detailed evaluation items for each capability are further presented, as shown in Fig. 3. Where items marked with "*" are the quantitative evaluation items whose quantized value directly reflects the evaluation results of the corresponding evaluation item, and unmarked items are the functional evaluation items whose evaluation results are reflected by whether to has the function of the corresponding item. The comprehensive evaluation results of teleoperation system are given by the combination of tasks requirements and comprehensive analysis of evaluation items. In addition to the function of hardware/software devices, the other main capabilities are indispensable for the space-oriented teleoperation system.

\section{Experiment Evaluation Method}

Teleoperation system is mainly developed for controlling space robot to fulfill various space tasks. To obtain a better experiment and system evaluation results, it's necessary to build tasks library of the teleoperation system to be evaluated and we should try our best to list as many specific tasks system can complete and detailed steps of each task as possible.

1) Data Acquisition of Specific Task(s): Based on the established tasks library, we must acquire the experimental data of at least one specific task for space robot. But data of one or a few specific task can only used for the performance evaluation of teleoperation system in a period or some aspects. Ideally, A large number of experiments covering all tasks should be carried out for the achievement of better experiment and system evaluation.

2) Experiment Evaluation: On the basis of the combination of the acquired experiment data and all quantitative and functional evaluation items shown in Fig. 3, the $i$-th $(i=1,2, \ldots, N)$ teleoperation experiment can be evaluated according to Eq. 1, and its evaluation result is indicated by $E_{i}$. Where assume that the $j$-th $(j=1,2, \ldots, M, M=10$ in this paper) universal capability summarized in section II-B1 has $m$ functional evaluation items and $n$ quantitative evaluation items.

$$
E_{i}=\sum_{i=1}^{M}\left(\boldsymbol{\alpha}_{j}^{\mathrm{T}} \mathbf{P}_{j}+\boldsymbol{\beta}_{j}^{\mathrm{T}} \mathbf{Q}_{j}\right)
$$

Where,

$$
\begin{aligned}
\boldsymbol{\alpha}_{j} & =\left[\alpha_{j 1}, \alpha_{j 2}, \ldots, \alpha_{j m}\right]^{\mathrm{T}} \\
\boldsymbol{\beta}_{j} & =\left[\beta_{j 1}, \beta_{j 2}, \ldots, \beta_{j n}\right]^{\mathrm{T}}
\end{aligned}
$$

and

$$
\begin{gathered}
\alpha_{j 1}+\alpha_{j 2}+\cdots+\alpha_{j m}+\beta_{j 1}+\beta_{j 2}+\cdots+\beta_{j n}=1 \\
\mathbf{P}_{j}=\left[p_{j 1}, p_{j 2}, \ldots, p_{j m}\right]^{\mathrm{T}} \\
\mathbf{Q}_{j}=\left[q_{j 1}, q_{j 2}, \ldots, q_{j n}\right]^{\mathrm{T}}
\end{gathered}
$$

and

$$
p_{j k}= \begin{cases}1, & \text { if } k \text {-th item is satisfied } \\ 0, & \text { otherwise }\end{cases}
$$




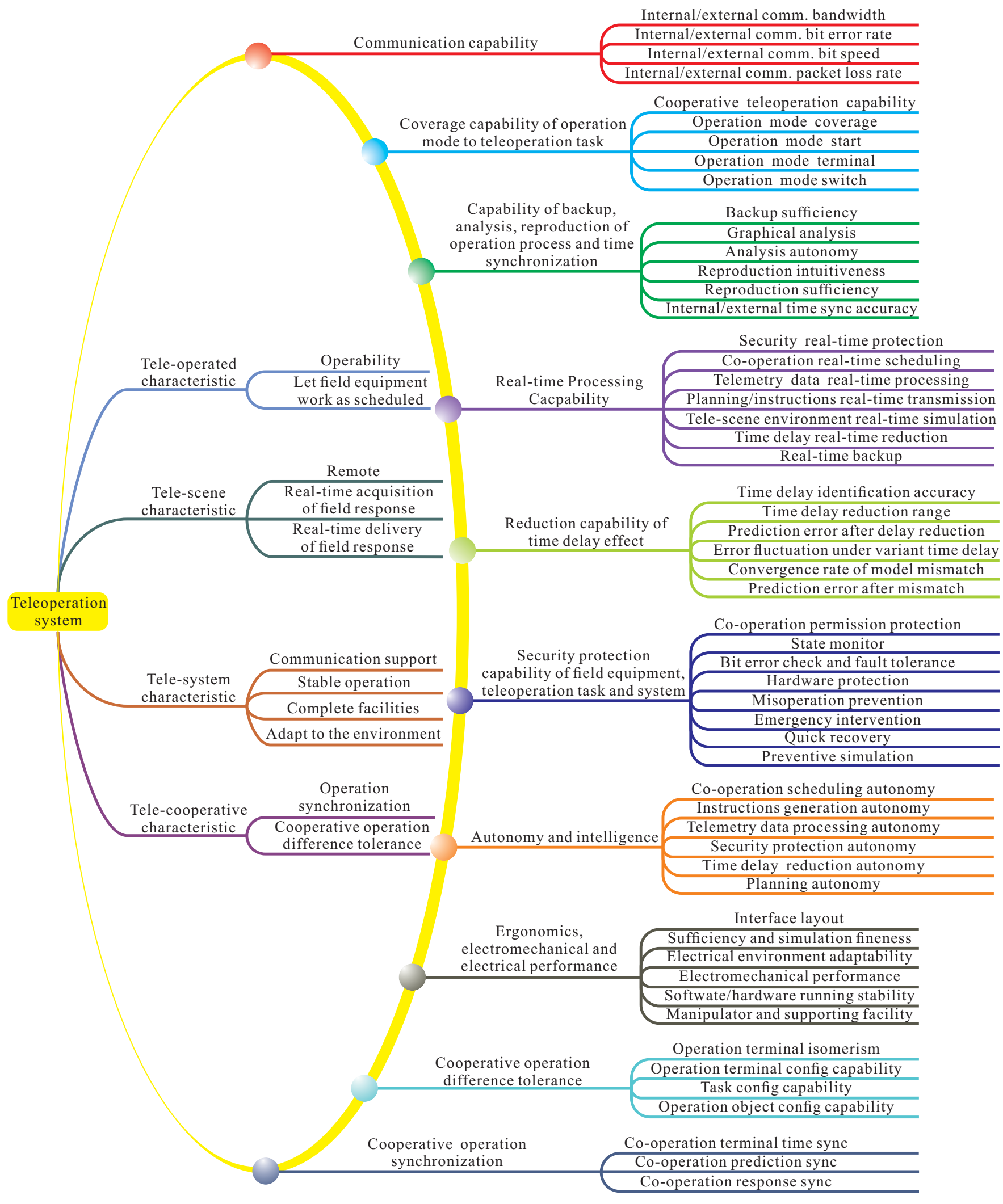

Fig. 2. Characteristics and universal capabilities of complete teleoperation system 


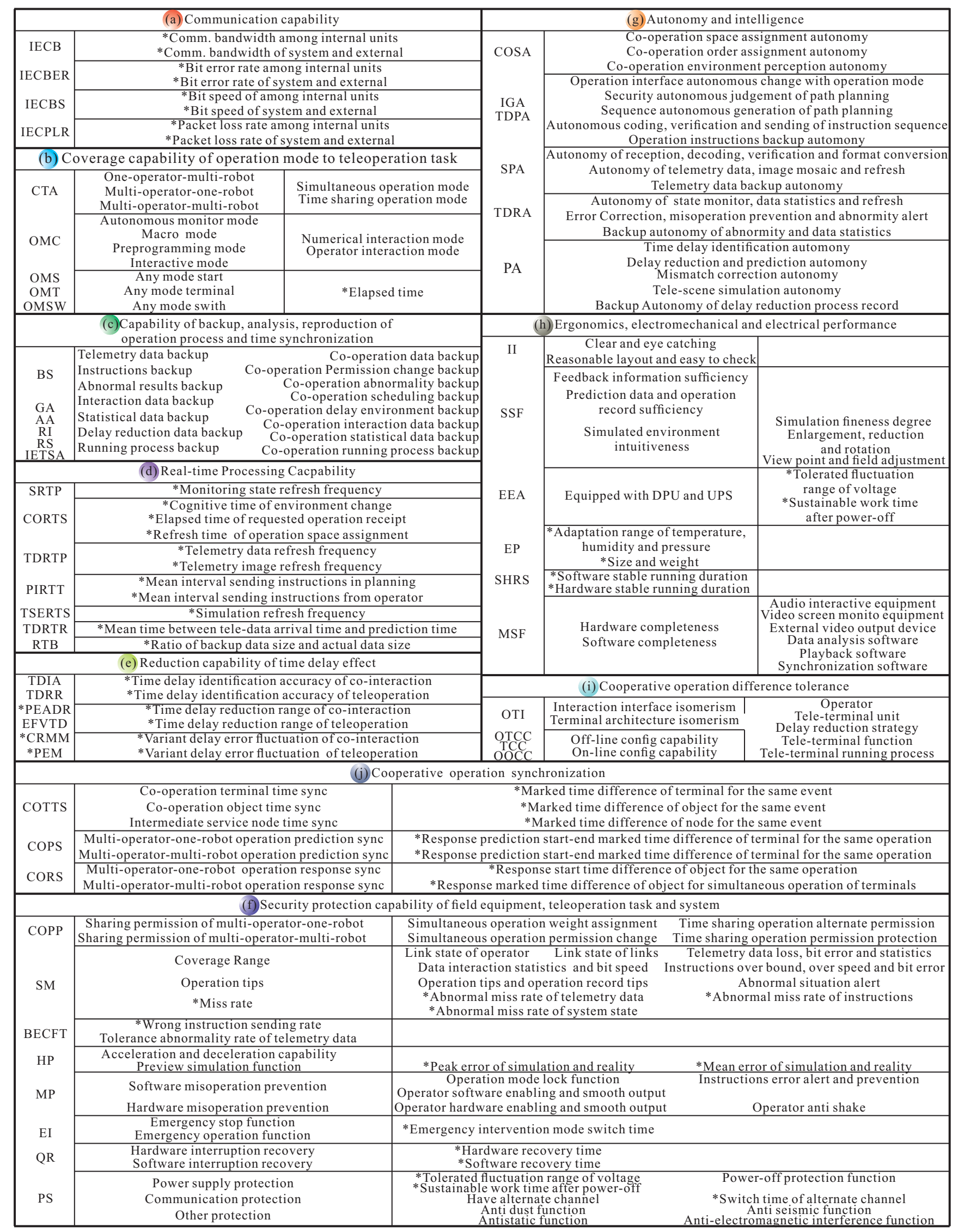

Fig. 3. Functional/quantitative evaluation items for universal capabilities (*quantitative evaluation item) 


$$
q_{j l}= \begin{cases}1, & \text { if } l \text {-th item is in target range } \\ 0, & \text { otherwise. }\end{cases}
$$

$\boldsymbol{\alpha}_{j}$ and $\boldsymbol{\beta}_{j}$ are weight matrix of functional and quantitative evaluation items respectively, and the value of their element $\alpha_{j k}(k=1,2, \ldots, m)$ and $\beta_{j l}(l=1,2, \ldots, n)$ is determined by the system characteristics interpreted in section II-A. $\mathbf{P}_{j}$ and $\mathbf{Q}_{j}$ are evaluation value of functional and quantitative evaluation items respectively, and the value of their element $p_{j k}(k=1,2, \ldots, m)$ and $p_{j l}(l=1,2, \ldots, n)$ is equal to 0 or 1 , determined by whether the criteria is satisfied.

Obviously, we can evaluate the $j$-th universal capability of $i$-th teleoperation experiment by the following equation.

$$
E_{i j}=\boldsymbol{\alpha}_{j}^{\mathrm{T}} \mathbf{P}_{j}+\boldsymbol{\beta}_{j}^{\mathrm{T}} \mathbf{Q}_{j}
$$

\section{System Evaluation Method}

1) Statistics of Evaluation Results: Before we give the comprehensive system evaluation, we first must complete evaluation of all $N$ experiment acquired in section II-C1 based on the experiment evaluation method Eq. 1. Then Statistics of the above experiment evaluation results can be further obtained.

2) Comprehensive System Evaluation: The result given by Eq. 1 is the evaluation result of an experiment, the comprehensive system evaluation can be defined as follows according the statistics results of $N$ experiments.

$$
E_{N}=\frac{\sum_{i=1}^{N} \sum_{i=1}^{M}\left(\boldsymbol{\alpha}_{j}^{\mathrm{T}} \mathbf{P}_{j}+\boldsymbol{\beta}_{j}^{\mathrm{T}} \mathbf{Q}_{j}\right)}{N}
$$

Where $E_{N}$ is the comprehensive evaluation result for teleoperation system.

Also, $E_{N j}$ can be taken as evaluation result of the $j$-th universal capability of the teleoperation system, and is defined by

$$
E_{N j}=\frac{\sum_{i=1}^{N}\left(\boldsymbol{\alpha}_{j}^{\mathrm{T}} \mathbf{P}_{j}+\boldsymbol{\beta}_{j}^{\mathrm{T}} \mathbf{Q}_{j}\right)}{N}
$$

\section{EVALUATION EXAMPLE}

To validate the proposed evaluation method, we designed a evaluation unit with Chinese interface displaying the acquired experiments data and evaluating universal capabilities mentioned above of our teleoperation system. The evaluation unit includes corresponding tabs of universal capabilities, each of which has user operation area, experiments data display area, evaluation items area and results display area and tab of comprehensive system evaluation for displaying comprehensive evaluation results, as shown in Fig. 4.

As shown in Fig. 5, it is the one of space robot joints angle in an teleoperation experiment of specific task; Fig. 6 is the corresponding joints angel error and we also display the realtime delay of this experiment in Fig. 7. Of course, there are lots of data about this experiment and other experiments we don't show here due to length limitation.

According to system capability evaluation Eq. 4, we obtain the capabilities evaluation results for our teleoperation system

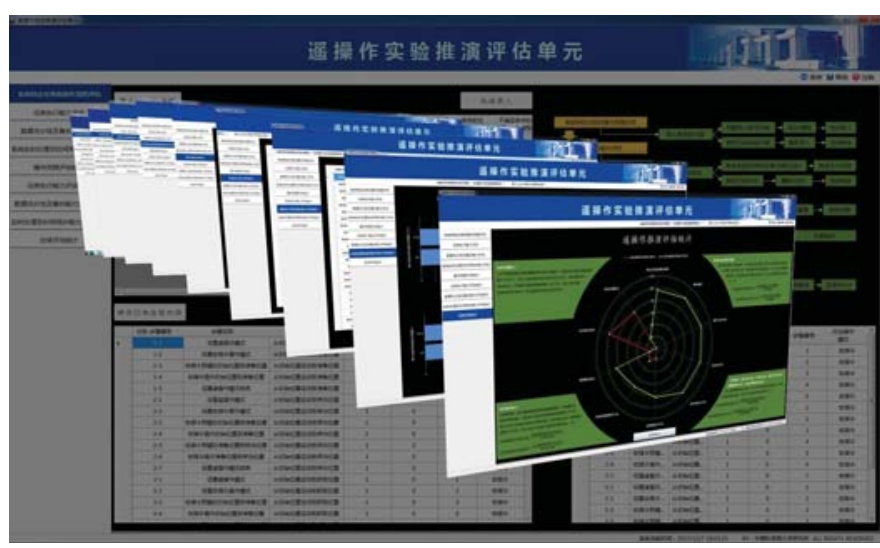

Fig. 4. Teleoperation system evaluation unit

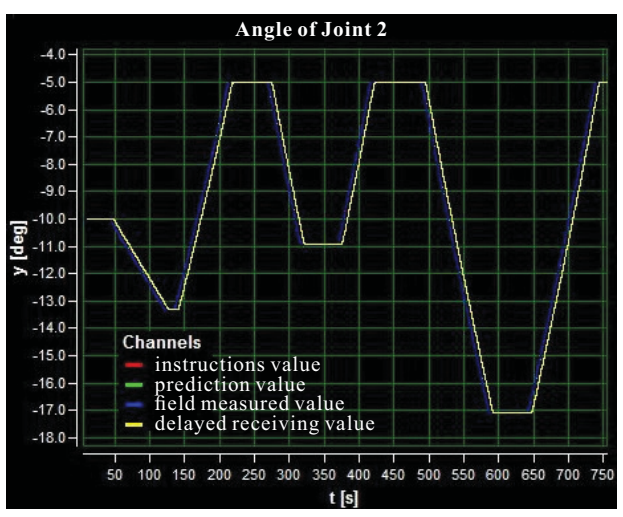

Fig. 5. Joint angle of an teleoperation experiment

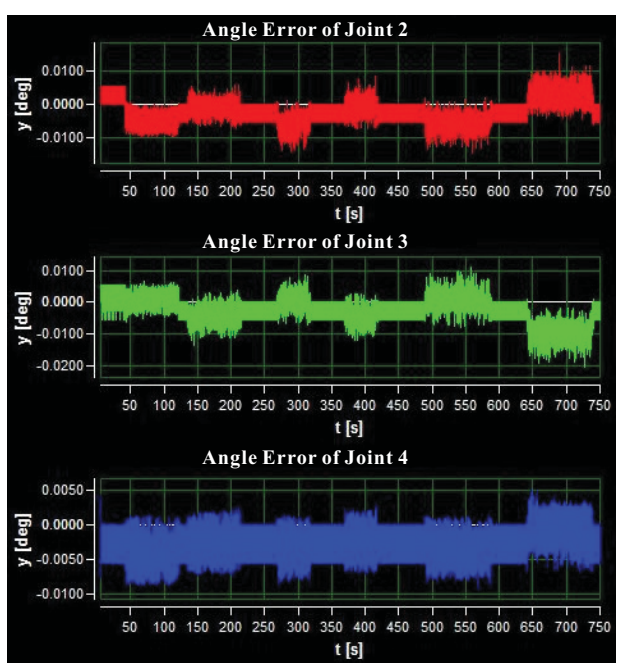

Fig. 6. Joint angle error

based on our acquired experiments data, shown in Fig. 8. Where $C_{i}(i=1,2, \ldots, 10)$ denotes the one of ten kinds of universal capabilities.

\section{CONCLUSION}

On the basis of detailed interpretation of four teleoperation system characteristics, ten universal capabilities of a complete 


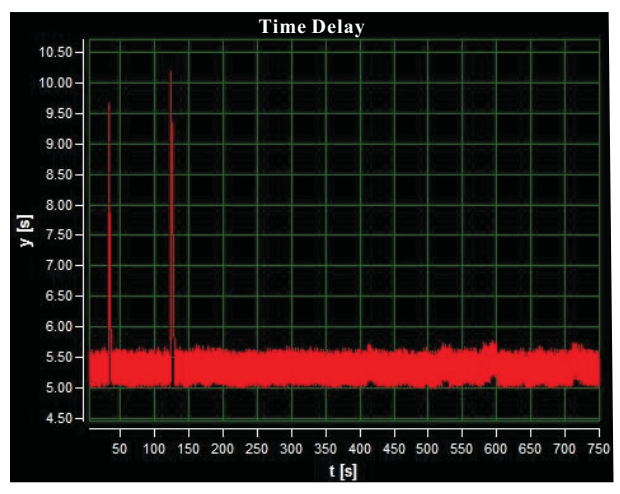

Fig. 7. Real-time delay

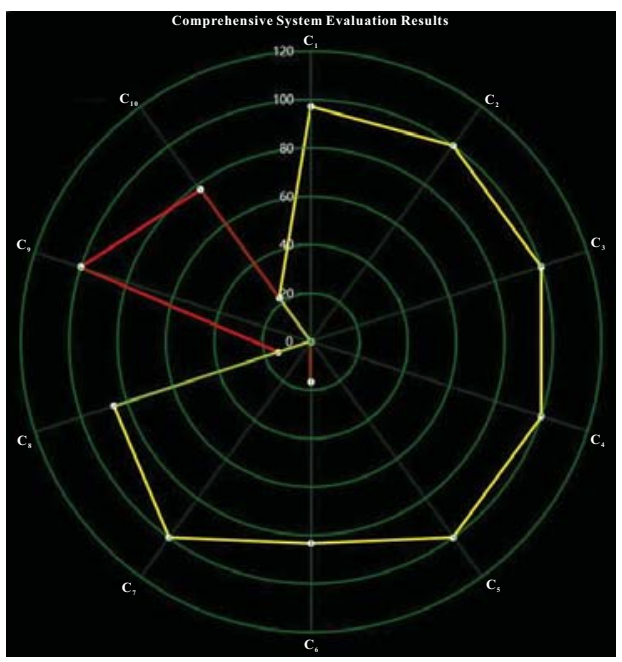

Fig. 8. Comprehensive teleoperation system evaluation results

teleoperation system were given in this paper; besides, 92 functional evaluation items and 49 quantitative evaluation items covering all capabilities were further summarized. Then a complete comprehensive evaluation method for space robot teleoperation experiment and system was proposed based on items, and the evaluation unit we designed and evaluation example we gave validated the proposed method, making up the study of teleoperation system evaluation and also positive to the development of tele-science technology.

\section{ACKNOWLEDGMENT}

This research was supported by the National Natural Science Foundation of China (grant number 11702294, 11002143); and the Key Project of Chinese National Programs for Fundamental Research and Development, 973 program (grant number 2013CB733000).

\section{REFERENCES}

[1] D. Feth, B. A. Tran, R. Groten, A. Peer, and M. Buss, Shared-Control Paradigms in Multi-Operator-Single-Robot Teleoperation. Springer Berlin Heidelberg, 2009.

[2] C. Yang, X. Wang, Z. Li, Y. Li, and C. Y. Su, "Teleoperation control based on combination of wave variable and neural networks," IEEE Transactions on Systems Man \& Cybernetics Systems, vol. PP, no. 99, pp. 1-12, 2016.
[3] T. Miyoshi, T. Imamura, and K. Terashima, "Design method of multilateral tele-control for multi-client and multi-coupled physical model server," in Advanced Robotics and ITS Social Impacts, 2013, pp. 206-211.

[4] M. Panzirsch, J. Artigas, J. H. Ryu, and M. Ferre, "Multilateral control for delayed teleoperation," in International Conference on Advanced Robotics, 2013, pp. 1-6.

[5] D. Powell and M. K. O’Malley, "The task-dependent efficacy of sharedcontrol haptic guidance paradigms," IEEE Transactions on Haptics, vol. 5 , no. 3, pp. 208-219, 2012

[6] P. Malysz and S. Sirouspour, "Trilateral teleoperation control of kinematically redundant robotic manipulators," International Journal of Robotics Research, vol. 30, no. 13, pp. 1643-1664, 2011.

[7] B. Khademian, "Control of dual-user teleoperation systems design, stability analysis, and performance evaluation," 2010. 